\title{
Cognitive Effects of Repeated Acute Exposure to Very High Altitude Among Altitude-Experienced Workers at $5050 \mathrm{~m}$
}

\author{
Matiram Pun, ${ }^{1,2}$ Veronica Guadagni, ${ }^{1-4}$ Lauren L. Drogos, ${ }^{1,2}$ Charlotte Pon, ${ }^{5}$ Sara E. Hartmann, ${ }^{1,2}$ \\ Michael Furian, ${ }^{6}$ Mona Lichtblau, ${ }^{6}$ Lara Muralt, ${ }^{6}$ Patrick R. Bader, ${ }^{6}$ Fernando A. Moraga, Daniel Soza, ${ }^{5}$ \\ Ivan Lopez, ${ }^{5}$ Jean M. Rawling, ${ }^{8}$ Silvia Ulrich, ${ }^{6}$ Konrad E. Bloch, ${ }^{6}$ Barry Giesbrecht, ${ }^{9}$ and Marc J. Poulin ${ }^{1-4,10,11}$
}

\begin{abstract}
Pun, Matiram, Veronica Guadagni, Lauren L. Drogos, Charlotte Pon, Sara E. Hartmann, Michael Furian, Mona Lichtblau, Lara Muralt, Patrick R. Bader, Fernando A. Moraga, Daniel Soza, Ivan Lopez, Jean M. Rawling, Silvia Ulrich, Konrad E. Bloch, Barry Giesbrecht, and Marc J. Poulin. Cognitive effects of repeated acute exposure to very high altitude among altitude-experienced workers at 5050 m. High Alt Med Biol. 20:361-374, 2019.

Background: We investigated altitude effects on different cognitive domains among perennial shift-workers at the Atacama Large Millimeter/submillimeter Array Observatory $(5050 \mathrm{~m})$, Chile.

Materials and Methods: Twenty healthy male workers were recruited and assigned to either a moderate-altitude first (MAF group, Test 1:2900 m and Test 2: $5050 \mathrm{~m}$ ) or to a high-altitude first (HAF group, Test 1:5050 m and Test 2: $2900 \mathrm{~m})$. Test 1 was conducted at the beginning and Test 2 at the end of the shift-work week. Processing speed (RTI, reaction time), attention (AST, attention-switching task, and RVP, rapid visual processing), and executive function (OTS, One Touch Stockings of Cambridge) were assessed.

Results: Of the three cognitive domains assessed, only processing speed showed altitude-at-test group interaction (RTI median five choice reaction time: $F_{1,17}=6.980, \eta_{p}^{2}=0.291, p=0.017$ ). With acclimatization, there was a decrease in AST reaction latency mean $\left(t_{17}=-2.155, d_{\mathrm{z}}=1.086, p=0.046\right)$, an increase in RVP accuracy $\left(t_{17}=2.733, d_{\mathrm{z}}=1.398, p=0.014\right)$, and a decrease in OTS mean latency first choice $\left(t_{17}=-2.375, d_{\mathrm{z}}=1.211\right.$, $p=0.03$ ). Decreased variability in cognitive function was observed in AST reaction latency standard deviation $\left(t_{17}=-2.524, d_{\mathrm{z}}=1.282, p=0.022\right)$ and in RVP response latency standard deviation $\left(t_{17}=-2.35, d_{\mathrm{z}}=1.177\right.$, $p=0.03$ ) with acclimatization. At $5050 \mathrm{~m}$ of elevation, $\mathrm{SpO}_{2}$ was positively correlated with executive function in the $M A F$ group (OTS problems solved on first choice: $r_{(5)}=0.839, p=0.018$ ) and negatively correlated with executive function latency standard deviations in the $H A F$ group (OTS latency to first choice standard deviation: $r_{(10)}=-0.618, p=0.032$ ).

Conclusions: Our findings highlight the importance of acclimatization and improvement of blood oxygen level, even among high altitude-experienced workers, to optimize performance of cognitively demanding work and reduce high altitude-associated health risks.
\end{abstract}

Keywords: acclimatization; ALMA; CANTAB; cognition; high altitude; hypoxia; workers

\footnotetext{
${ }^{1}$ Department of Physiology and Pharmacology, Cumming School of Medicine, University of Calgary, Calgary, Canada.

${ }^{2}$ Hotchkiss Brain Institute, Cumming School of Medicine, University of Calgary, Calgary, Canada.

${ }^{3}$ Department of Clinical Neuroscience, Cumming School of Medicine, University of Calgary, Calgary, Canada.

${ }^{4}$ O'Brien Institute for Public Health, Cumming School of Medicine, University of Calgary, Calgary, Canada.

${ }^{5}$ Safety Group, Atacama Large Millimeter Submillimeter Array, Calama, Chile.

${ }^{6}$ Department of Respiratory Medicine, Sleep Disorders Centre and Pulmonary Hypertension Clinic, University Hospital Zurich, Zurich, Switzerland.

${ }^{7}$ Laboratorio de Fisiología, Hipoxia y Función Vascular, Departamento de Ciencias Biomédicas, Facultad de Medicina, Universidad Católica del Norte, Coquimbo, Chile.

${ }^{8}$ Department of Family Medicine, Cumming School of Medicine, University of Calgary, Calgary, Canada.

${ }^{9}$ Department of Psychological and Brain Sciences, and Institute for Collaborative Biotechnologies, University of California Santa Barbara, Santa Barbara, California.

${ }^{10}$ Libin Cardiovascular Institute of Alberta, Cumming School of Medicine, University of Calgary, Calgary, Canada.

${ }^{11}$ Faculty of Kinesiology, University of Calgary, Calgary, Canada.
} 


\section{Introduction}

W HEN HUMANS ARE EXPOSED to high-altitude environments, the reduced barometric pressure at altitude leads to a reduction in the amount of oxygen in the body, also known as hypoxia. The brain is highly sensitive to hypoxia. With altitude exposure, a number of brain functions are impaired (Wilson et al., 2009; McMorris et al., 2017). Symptoms associated with hypoxia can be various, but often include headache, dizziness, fatigue, and neurocognitive impairment, which may manifest early on during the altitude exposure (Wilson et al., 2009). The magnitude of hypoxic impairment depends upon the rate of ascent and absolute altitude gained (Bjursten et al., 2010; Maa, 2010).

Shift-workers at the Atacama Large Millimeter/submillimeter Array (ALMA) Observatory $(5050 \mathrm{~m})$ on the Chajnantor Plateau, Chile, are exposed to high altitude during alternate weeks in their work cycle (Moraga et al., 2018). Typically, the workers quickly ascend to a moderate altitude on the first day of their shift, traveling from Santiago $(520 \mathrm{~m})$ to the Operation Support Facility of ALMA (OSF, $2900 \mathrm{~m}$ ). On the second day, they reach the work station (i.e., ALMA Observatory, $5050 \mathrm{~m}$ ). Throughout the shift-work week, the workers sleep at OSF $(2900 \mathrm{~m})$ and commute by motor vehicle ( $\sim 45$ minutes) to work at the ALMA Observatory $(5050 \mathrm{~m})$. At the end of the week, the workers return to near sea level at Santiago $(520 \mathrm{~m})$ for a week of rest.

Previous studies have shown that acute exposure to very high altitude $(5050 \mathrm{~m})$ leads to profound hypoxemia (near sea level $\mathrm{SpO}_{2}: 98.0 \% \pm 0.9 \%$ to altitude $\mathrm{SpO}_{2}: 80.2 \% \pm 4.7 \%$ ), which improves with acclimatization over a week $\left(\mathrm{SpO}_{2}\right.$ at $5050 \mathrm{~m}: 83.7 \% \pm 4.5 \%$ ), although individuals tend to remain significantly hypoxemic when compared to sea level measurements (Pun et al., 2018b). The acute hypoxemia associated with brain tissue hypoxia at these altitudes can adversely affect cognitive function such as attention (Limmer and Platen, 2018; Pun et al., 2018a), executive functions (Asmaro et al., 2013; Ochi et al., 2018a, b), information processing (Davranche et al., 2016), and memory (Kramer et al., 1993).

High-altitude exposure has both acute and chronic effects on the human brain (Hornbein, 2001). Alterations in both brain structure and vasculature are hypothesized to underlie the cognitive deficits that result from chronic exposure to high-altitude hypoxia-attributed hypoxemia (Cramer et al., 2019). Previously, we examined the effects of acute exposure to high altitude in altitude-naive individuals traveling to the ALMA observatory (Pun et al., 2018a, b). We reported that these individuals made more mistakes, had slower reaction times (RTIs), and exhibited decreased sustained attention when at high altitude.

The high-altitude workers at the ALMA Observatory $(5050 \mathrm{~m})$ repeatedly experience periodic high-altitude hypoxia for several years. Only one other study (Moraga et al., 2018) has investigated the cognitive effects of high-altitude exposure in ALMA workers. This research specifically examined constructive praxis and visuospatial memory through the use of the Folstein Mini-Mental State Examination Test and the Rey-Osterrieth Complex Figure Test (Moraga et al., 2018). The results demonstrated that cognitive function was impaired at high altitude, but that it improved following oxygen supplementation. A previous study, performed in normobaric hypoxic conditions, included a short-term preacclimatization period and observed reduced frequency of risky decision-making compared to a sham (control) condition of acclimatization (Niedermeier et al., 2017). Thus, cognitive function may be affected differently by initial altitude exposure, whether moderate or high altitude, and over the course of the acclimatization.

While many studies have investigated the acute effects of hypoxia on cognitive functioning, only a few studies included high-altitude shift-workers. The workers at the ALMA observatory are mostly engaged in jobs that require high levels of focus, dexterity, and sustained attention. The weekly shift schedule that they follow is supposed to minimize the negative impact of high altitude exposure; however, findings from previous studies do not show beneficial effects of this work schedule and further investigations using comprehensive cognitive batteries are required.

In this study, we investigated the effects of acute highaltitude exposure and acclimatization on cognitive performance in altitude-experienced ALMA workers, over a week spent between the OSF (2900 m) and ALMA (5050 m). We hypothesized that experienced ALMA workers would show impaired cognitive functioning during acute exposure to high altitude. These cognitive changes at altitude would be associated with decreased blood oxygen saturation, and would be inversely correlated with years of work experience at ALMA. Finally, we hypothesized that cognitive function would improve with acclimatization during the shift-work week.

\section{Materials and Methods}

\section{Participants}

The participants were recruited at the OSF $(2900 \mathrm{~m})$ when they were on the shift-work schedule at ALMA. The recruitment was done at the beginning of the shift-work at ALMA (i.e., on the day of arrival at the OSF). Participants were enrolled by use of advertisement flyers and by word of mouth from the local study investigators who were fluent in both Spanish and English. ALMA workers recruited for the study were low-altitude permanent residents (sea level and near sea level) who had been doing shift-work at ALMA for about 1 year or longer. Once the workers agreed to participate in the study, they met with the local study investigators to review the study procedures, to ask any questions about the study, and to provide informed written consent. After the recruitment, baseline measurements and a medical history questionnaire were completed. ALMA workers had already been screened to work at very high altitude $(5050 \mathrm{~m})$ during their employment process and underwent periodic health check-ups by the medical team of ALMA. Thus, no additional screening was completed for this study. After the completion of the baseline measurement and medical history, the workers were assigned to either moderate altitude first $(M A F)$ group or to high altitude first $(H A F)$ group as described in further detail below (see Study Design section). Details of the baseline measurements and medical history of the study participants are presented in Table 1.

\section{Study design}

Participants were assigned to two groups. One group completed the first cognitive assessment at the OSF (2900 m) and was therefore called the $M A F$ group. The second group completed the first cognitive assessment at the Observatory $(5050 \mathrm{~m})$ and was called the $H A F$ group. This first test was 


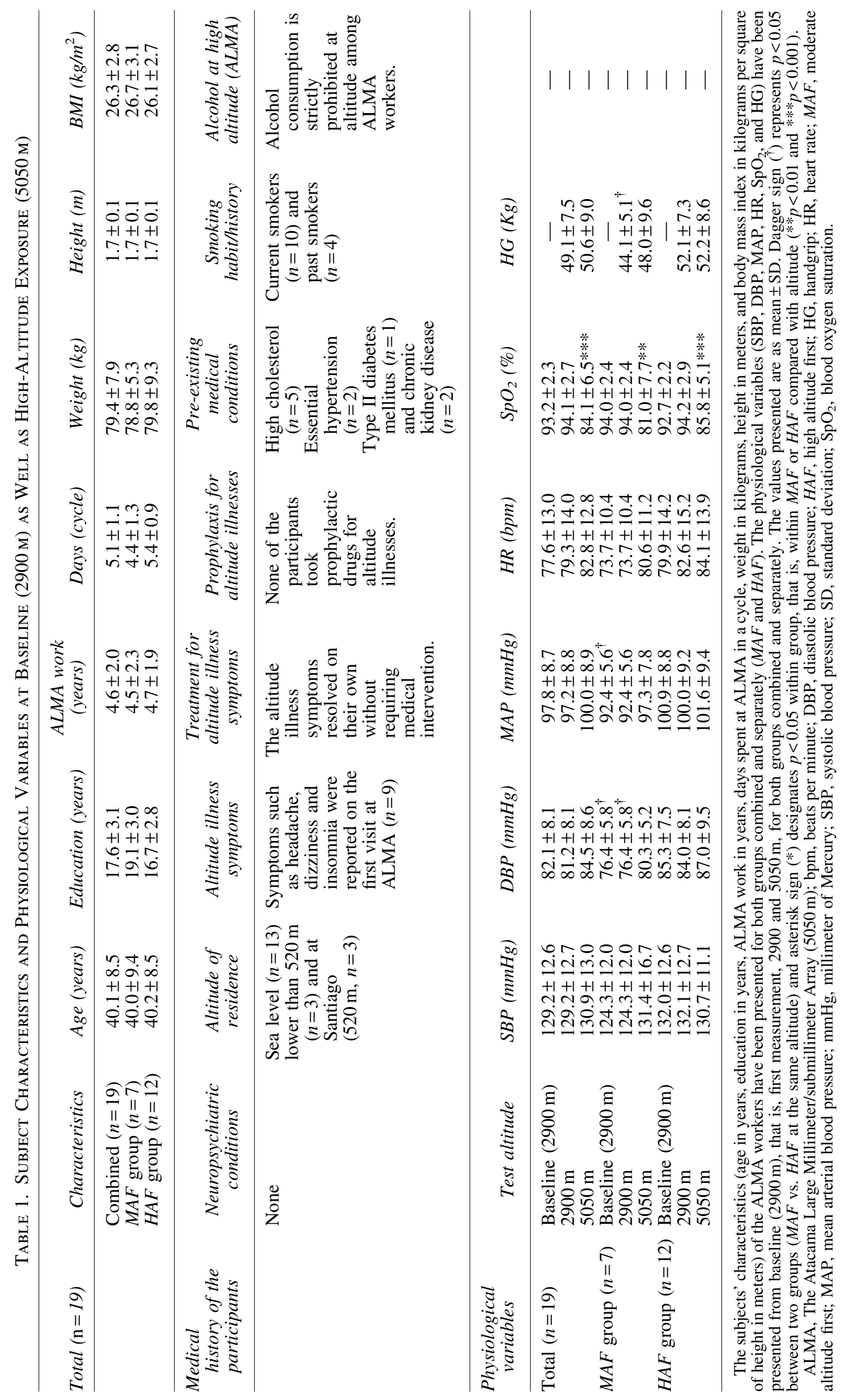


done on the first day of arrival at the worksite: at OSF $(2900 \mathrm{~m})$ for the $M A F$ group and at ALMA $(5050 \mathrm{~m})$ for the $H A F$ group. The second cognitive assessment was done at the end of the work-shift, (i.e., after $5.1 \pm 1.1$ days of repeated ascent to ALMA for both groups, but at the inverse location). The cognitive test at OSF was done early in the morning (810 AM), while the test at ALMA was done between 12 and 2 PM. Blood pressure was recorded during rest with an automatic arm blood pressure monitor (Omron Healthcare, Inc.). Measurements were taken a few hours after arrival at altitude and in a comfortable sitting position. Blood oxygen saturation and heart rate were measured with a finger pulse oximeter. Handgrip strength was measured using a strain-gauge dynamometer (Lafayette Instrument Co.) in the dominant hand and the peak strength was reported.

There were 12 participants in the $H A F$ group and 8 in the $M A F$ group. One participant could not complete the tests in the $M A F$ group because of reasons unrelated to the experiment. Therefore, data for 19 participants $(H A F: 12$ and $M A F: 7)$ were analyzed. The study flow chart with the altitude exposure as well as the cognitive assessment schedule is illustrated in Figure 1A and B. The study was approved by the University of Calgary Conjoint Health Research Ethics Board (CHREB ID: REB15-2709). All participants provided informed written consent before starting the experiment.

\section{Cognitive test battery}

The cognitive battery used in the study was a custom battery assembled with tests chosen from the Cambridge Neuropsychological Test Automated Battery (CANTAB) collection (CANTAB, 1994). The same custom battery was previously used by our group (Pun et al., 2018a). These batteries are robust, validated to be administered in multiple languages and can be utilized several times without learning and repetition effects (Lowe and Rabbitt, 1998; Syvaoja et al., 2015). We selected tests to assess three cognitive domains: processing speed, sustained attention, and executive functions. Processing speed was measured with the RTI task. This task measures movement time, RTI, accuracy, and impulsivity. The outcomes considered in the analyses were the RTI median fivechoice reaction time (RTIFMDRT), RTI mean five-choice movement time (RTIFMMT), and RTI five-choice movement time standard deviation (RTIFMTSD). Sustained attention and inhibition were assessed with the attention switching tasks (AST) and rapid visual processing (RVP) task. The AST is a task measuring top-down control and inhibition of irrelevant information. The outcomes analyzed were the AST total correct (ASTTC) score as a measure of accuracy, the AST reaction latency mean (ASTLM) as a measure of RTI, and the AST reaction latency standard deviation (ASTLSD) as a measure of sensitivity to target. Similarly, the RVP measures RTI, accuracy, and target sensitivity. The RVP Accuracy (RVPA), mean response latency (RVPMDL), and the RVP response latency standard deviation (RVPLSD) were the outcomes considered in the analyses. Executive functions were assessed with the One Touch Stockings of Cambridge (OTS) task, which assesses spatial planning ability. Analyzed outcomes include the OTS problems solved on first choice (OTSPSFC), the OTS mean latency to first choice (OTSMLFC), and the OTS latency to first choice standard deviation (OTSLFCSD). Participants completed the cognitive battery in 30 minutes; the order of the specific tests was automatically randomized. The cognitive assessment was performed using the CANTAB Connect Research Suite (Cambridge Cognition, CANTAB ${ }^{\circledR}$ Cognitive Assessment Software) for iPad Air 1 (model: A1474, dimensions: 9.7 inches retina display, iOS 9.3.1, Apple, Inc.) and was completed in 30 minutes.

The cognitive batteries were administered in a warm, wellventilated, and bright dedicated room in the ALMA facilities (OSF at $2900 \mathrm{~m}$ and ALMA at $5050 \mathrm{~m}$ ). The humidity was around $20 \%$, and temperature was maintained at $\sim 22^{\circ} \mathrm{C}$ in the experimental rooms. The cognitive assessment was conducted in identical setting at both locations, except at different altitudes. The study participants were requested to follow their usual routine activities, physical activity, and ALMA work schedule during the entire period of the study.

\section{Data analyses}

We performed two corrections to isolate the effect of high altitude at ALMA $(5050 \mathrm{~m})$ and acclimatization at OSF $(2900 \mathrm{~m})$ without the confounding effects of test order (repetition) and very high-altitude exposure (ALMA, $5050 \mathrm{~m}$ ), respectively. These corrections were necessary due to the fact that altitude-at-first-test and altitude were not manipulated factorially (i.e., fully crossed). The first correction was implemented to counterbalance the effect of cognitive test order, thus isolating the independent effect of very high altitude on cognition. The second correction removed the effect of $5050 \mathrm{~m}$ exposure, to explore the effects of the sleeping altitude (OSF, $2900 \mathrm{~m}$ ) on cognitive performance. Specific details of these calculations have been described in the subsections below.

High-altitude effect (ALMA, 5050 m) on cognitive function. We first arranged the data by the test schedule, that is, Test 1 and Test 2 for each cognitive outcome, including both groups. We then computed the mean $(M)$ score for Test 1 across both groups $\left(M_{\text {Test } 1}\right)$, as well as the mean score for Test 2 across both groups $\left(M_{\text {Test } 2}\right)$. We computed a change score by subtracting the mean scores on Test 2 from the mean score on Test 1 $\left(M_{\text {Test1 }}-M_{\text {Test } 2}\right)$. This computed change score represents the test repetition effect. We then subtracted this change score from the scores on Test 1 to correct for the potential test repetition effect [i.e., Test $1-\left(M_{\text {Test } 1}-M_{\text {Test } 2}\right)=$ Test $1-$ Corrected

FIG. 1. Study flowchart for $M A F$ and $H A F$ group study participants with their testing sessions. (A) Flowchart of the study participants from $M A F$ and $H A F$ group with baseline clinical measurements and cognitive tests (Test 1 and Test 2). (B) Illustration of cognitive testing of both groups $(M A F$ and $H A F)$ with altitude exposure. $Y$-axis represents altitude and $X$ axis expedition in days in a typical cycle. The dashed line represents the testing schedule of the $M A F$ group with the altitude gain, while the solid line represents testing the schedule of the HAF group participants. ALMA, the Atacama Large Millimeter Array; ASOF, ALMA Support Operation Facility; HAF, High Altitude First; HAF Test 1, first test for highaltitude group; $H A F$ Test 2, second test for high altitude; $M A F$, moderate altitude first; $M A F$ Test 1 , first test for moderate altitude; MAF Test 2, second test for moderate altitude group. 


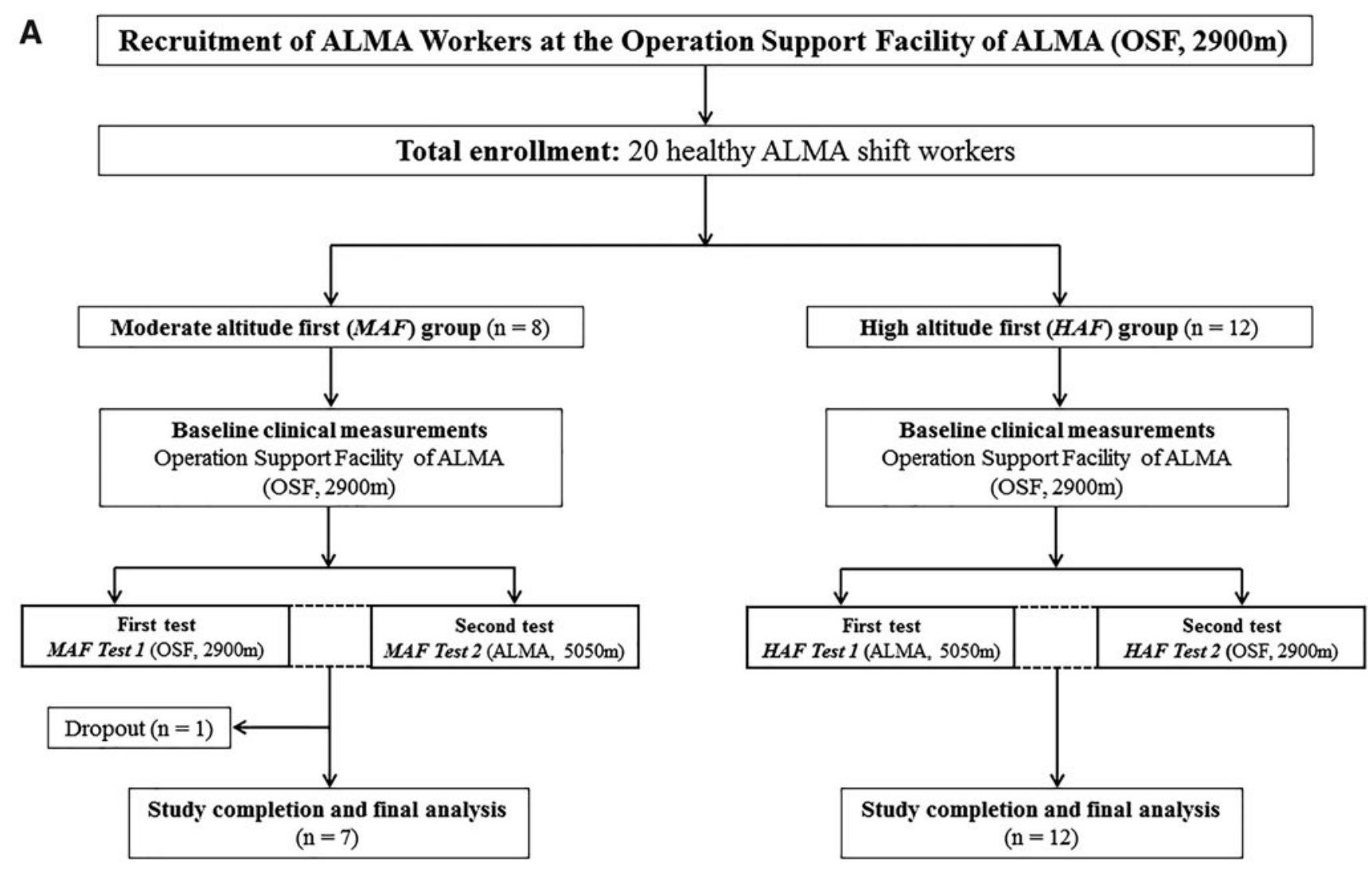

告)

B

Cognitive test

Cognitive test

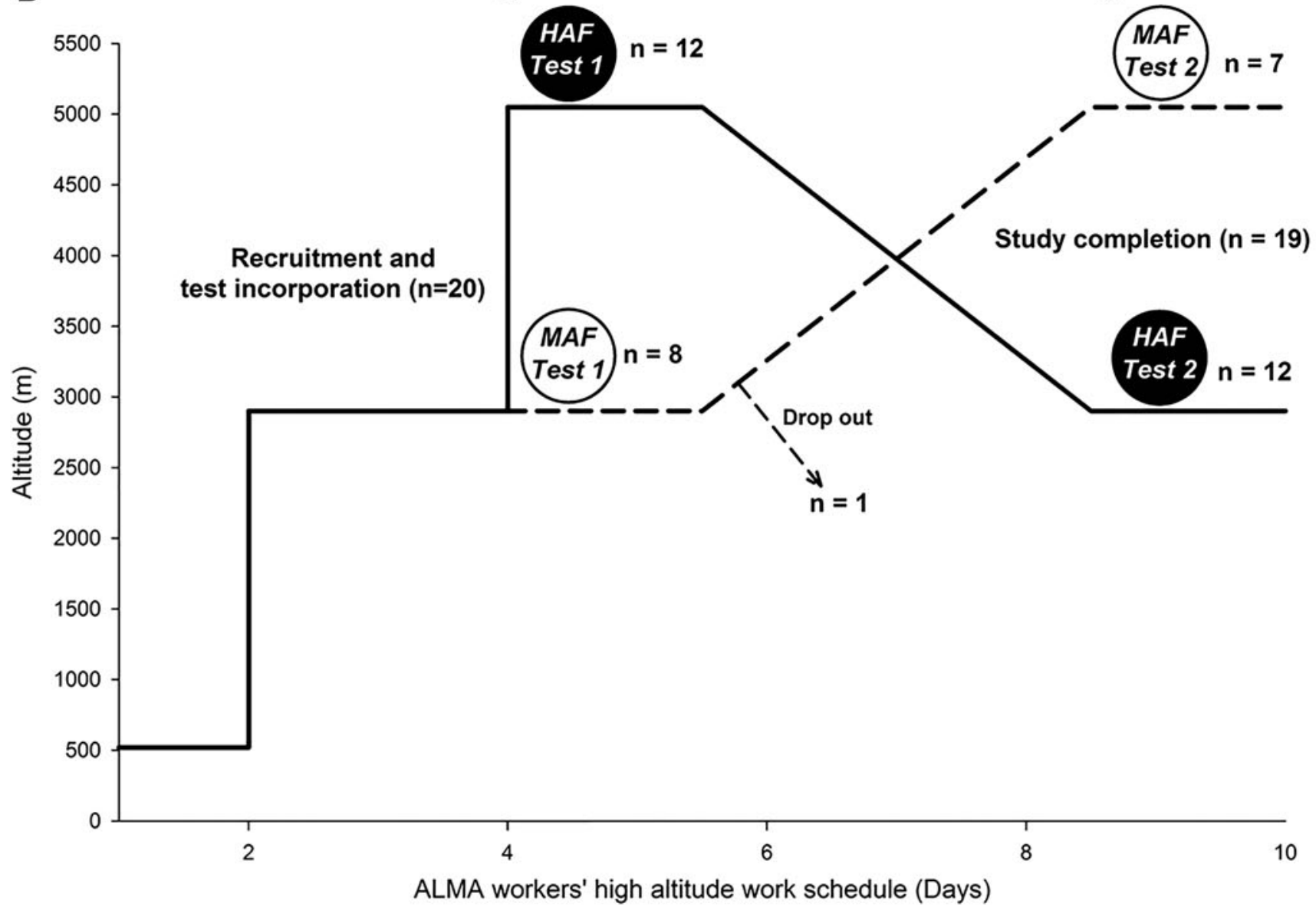


Scores]. This procedure was used for all cognitive outcomes and the corrected values were used for subsequent statistical analyses unless otherwise stated.

Mixed model repeated measures analyses of variance. We ran multiple mixed model repeated measures analyses of variance (RM ANOVAs) on all cognitive outcomes using altitude-at-test cognitive scores (Test 1 -Corrected Scores vs. Test 2 Scores) as a within-subjects factor, and Group (starting Altitude Group: MAF2900 vs. HAF5050) as a betweensubjects factor. Significant interactions were further explored with simple effects test (pairwise comparisons).

Effects of high-altitude acclimatization on cognitive functions. The high-altitude acclimatization was explored at two elevations (i.e., OSF-2900 m, and ALMA-5050 m). Since the ALMA workers were tested at the beginning and end of their schedules (average cycle duration: 5.1 \pm 1.1 days), the acclimatization effect of high-altitude exposure was explored comparing the scores on the first test (Test 1) versus second test (Test 2) of the respective altitude between two groups with an independent samples $t$-test. The acclimatization at the OSF (2900 m) was completed comparing scores for the MAF Test 1 (Day 1) versus HAF Test 2 (Day 5). Similarly, the acclimatization effect at ALMA $(5050 \mathrm{~m})$ was tested comparing $H A F$ Test 1 (Day 1) versus MAF Test 2 (Day 5).

Cognition as a function of sleeping altitude (OSF, $2900 \mathrm{~m}$ ). We further explored the effects of sleeping altitude (OSF, $2900 \mathrm{~m}$ ) on the cognitive outcomes by removing the confounding effect of very high-altitude exposure (ALMA, $5050 \mathrm{~m})$. Here, we computed the mean $(M)$ score for Test 1 in the $M A F$ group $\left(M_{\text {Test } 1} M A F\right)$ and the mean score for Test 1 in the $H A F$ group $\left(M_{\text {Test } 1} H A F\right)$, and then computed a change score subtracting the mean scores on Test 1 for the $M A F$ group from the mean scores on Test 1 for the $H A F$ group $\left(M_{\text {Test } 1}\right.$ $\left.H A F-M_{\text {Test } 1} M A F\right)$. We then subtracted this change score from the scores on Test 1 for the $H A F$ group [i.e., Test $1 H A F-\left(M_{\text {Test } 1}\right.$ $\left.H A F-M_{\text {Test } 1} M A F\right)=$ Test $1 H A F-$ Corrected Scores $]$ and from the scores on Test 2 for the MAF group [i.e., Test $2 M A F-$ $\left(M_{\text {Test } 1} H A F-M_{\text {Test } 1} M A F\right)=$ Test $2 M A F-$ Corrected Scores $]$ to correct for the acute effect of very high altitude, while centering all scores to the moderate altitude, that is, OSF (2900 m). This procedure was used for all cognitive outcomes. A paired samples $t$-test was then used to compare "Test $1 H A F-C o r$ - rected Scores" versus "Test $2 H A F$ Scores" for the HAF group and "Test 1 MAF Scores" versus "Test $2 M A F$ - Corrected Scores" for the MAF group to test the presence of an acclimatization effect within groups at moderate altitude.

Hypoxemia and cognitive functions: Pearson's correlation. Even though the overall sample size was small, we had valid a priori reasons to expect associations between altitude exposure and cognitive functioning. To explore these associations between changes in cognitive function and response to altitude exposure, we used a Pearson correlation analysis to assess the linear association between cognitive scores at high altitude $(5050 \mathrm{~m})$, hypoxemia $\left(\mathrm{SpO}_{2}\right)$ as a measure of the acute altitude effect, and years of work experience at high altitude $(5050 \mathrm{~m})$.

All the data analyses were conducted with the Statistical Package for the Social Sciences (SPSS, Version 24, IBM Co.). SigmaPlot 14.0 (Systat Software, Inc.) was used to plot the data. The criterion for assessing statistical significance was set at $p<0.05$, two tailed. Bonferroni correction for multiple comparisons was used in the follow-up tests. The effect sizes for both ANOVA (partial eta squared, $\eta_{p}^{2}$ ) and $t$ tests (Cohen's $d, d_{\mathrm{z}}$ ) have been mentioned in the results.

\section{Results}

The two groups were similar in terms of age, education, work experience at ALMA (chronicity of periodic hypobaric hypoxia exposure), and number of days of exposure per cycle at high altitude $(5050 \mathrm{~m})$, as shown in Table 1 . The baseline mean arterial blood pressure was different between groups (MAF: $92.4 \pm 5.6 \mathrm{mmHg}$ vs. $H A F: 100.9 \pm 8.8 \mathrm{mmHg}, t_{17}=-2.291$, $\left.d_{\mathrm{z}}=1.154, p=0.035\right)$, which was attributed to differences in diastolic blood pressure (MAF: $76.4 \pm 5.8 \mathrm{mmHg}$ vs. $H A F$ : $\left.85.3 \pm 7.5 \mathrm{mmHg}, t_{17}=-2.692, d_{\mathrm{z}}=1.327, p=0.015\right)$. However, the second measurement (i.e., second mean arterial blood pressure at $2900 \mathrm{~m}$ ) was not significantly different between groups $\left(t_{17}=-1.972, d_{\mathrm{z}}=0.999, p=0.065\right)$, although diastolic blood pressure of $H A F$ group remained slightly higher $\left(t_{17}=-2.16\right.$, $\left.d_{\mathrm{z}}=1.075, p=0.045\right)$. Interestingly, the $H A F$ group had slightly higher handgrip strength at $2900 \mathrm{~m}(M A F: 44.1 \pm 5.1 \mathrm{Kg}$ vs. $\left.H A F: 52.1 \pm 7.3 \mathrm{Kg}, t_{17}=-2.559, d_{\mathrm{z}}=1.277, p=0.020\right)$. Both groups were significantly hypoxemic with exposure to high altitude $\left(\mathrm{SpO}_{2}\right.$ of $M A F: 94.0 \% \pm 2.4 \%$ vs. $81.0 \% \pm 7.7 \%$, $t_{6}=5.629, d_{\mathrm{z}}=2.128, p<0.01$ and $\mathrm{SpO}_{2}$ of $H A F: 94.2 \% \pm 2.9 \%$ vs. $\left.85.8 \% \pm 5.1 \%, t_{11}=7.008, d_{\mathrm{z}}=2.023, p<0.001\right)$.

FIG. 2. Absolute cognitive values from two testing points (2900 and $5050 \mathrm{~m})$ of both groups of ALMA workers during a work cycle. The figure illustrates absolute raw cognitive values during the two tests collected at the two altitude points $(2900$ and $5050 \mathrm{~m}$ ) for the two groups $(M A F$ and $H A F)$. The first panel (A-C) illustrates processing speed or RTI parameters (RTIFMDRT, RTIFMMT, and RTIFMTSD). The second box panel displays attention scores, with the first panel within the box (D-F) showing the AST parameters (ASTTC, ASTLM, and ASTLSD) and the second panel within the second box (G-I) showing RVP parameters (RVPA, RVPMDL, and RVPLSD). The third box panel (J-L) represents executive function, indicated by OTS parameters (OTSPSFC, OTSMLFC, and OTSLFCSD). The $X$-axis depicts cognitive testing time points, while the $Y$-axis depicts changes in cognitive parameters as mean \pm standard deviation for both $M A F$ and $H A F$ groups. The altitude in meters, noted at the testing time points, specifies the altitude at test. Symbols: Black solid lines, MAF group; black dotted lines, HAF group. Note: Cognitive functions are expressed in "ms" (milliseconds), except ASTTC and OTSPSFC, which are expressed in " $n$ " (numbers). Test 1, first cognitive test; Test 2, second cognitive test. AST, Attention Switching Task; ASTLM, AST reaction Latency Mean; ASTLSD, AST reaction Latency Standard Deviation; ASTTC, AST Total Correct; OTSLFCSD, One Touch Stockings of Cambridge Latency to First Choice Standard Deviation; OTSMLFC, One Touch Stockings of Cambridge Mean Latency First Choice; OTSPSFC, One Touch Stockings of Cambridge Problems Solved on First Choice; RTIFMDRT, RTI Median Five-choice Reaction Time; RTIFMMT, RTI Mean Five-choice Movement Time; RTIFMTSD, RTI Five-choice Movement Time Standard Deviation; RVPA, Rapid Visual Processing Accuracy; RVPLSD, RVP Response Latency Standard Deviation; RVPMDL, RVP Mean Response Latency. 
Processing speed Reaction Time (RTI)

A

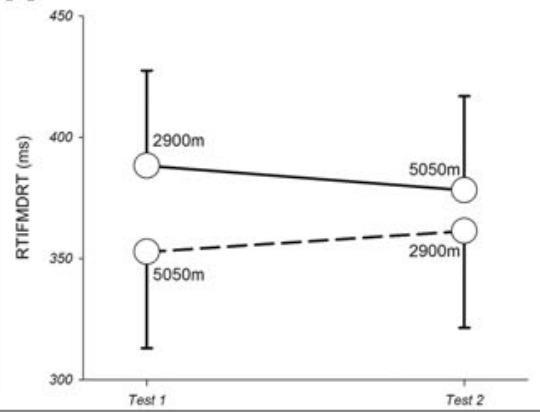

Moderate Altitude First (MAF) starts at $2900 \mathrm{~m}$

B

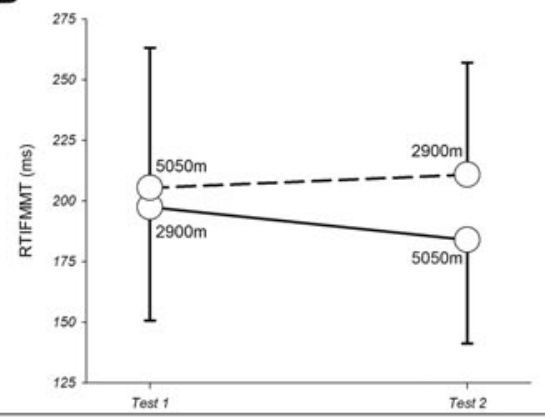

C

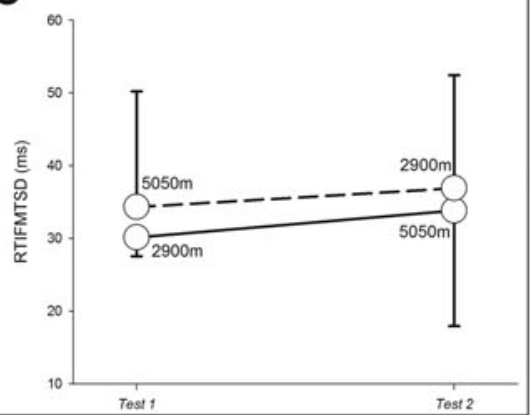

\section{Attention}

Attention Switching Task (AST)

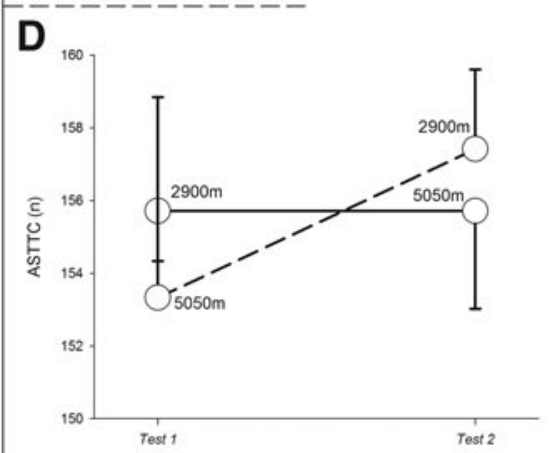

E

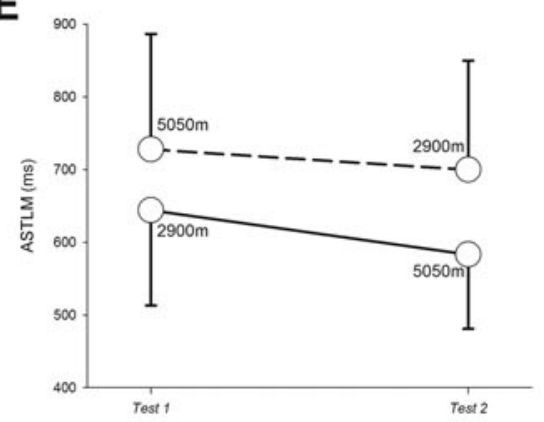

H

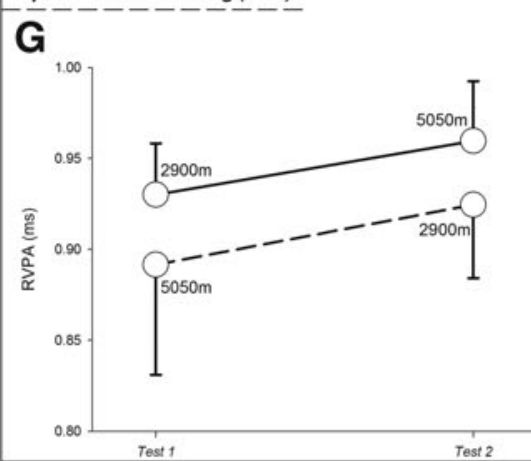

$\mathbf{F}$

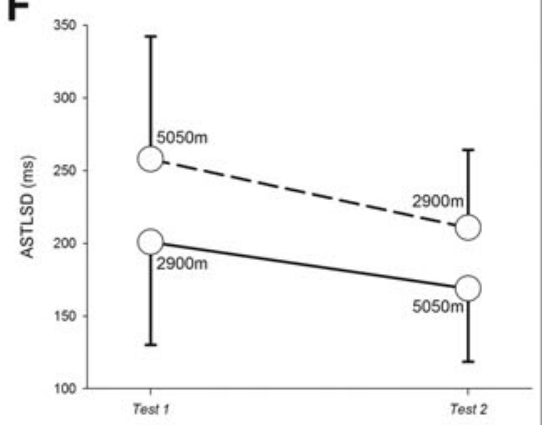

I

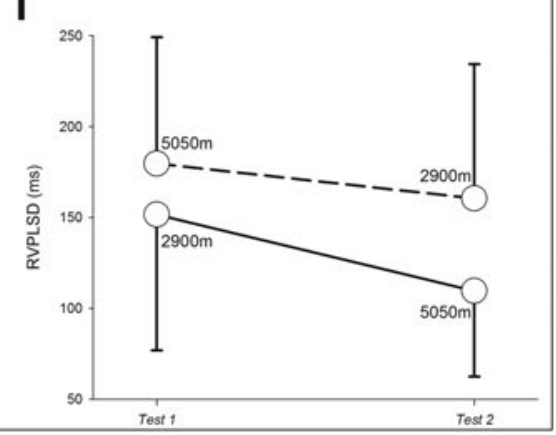

Executive function

One Touch Stockings of Cambridge (OTS)

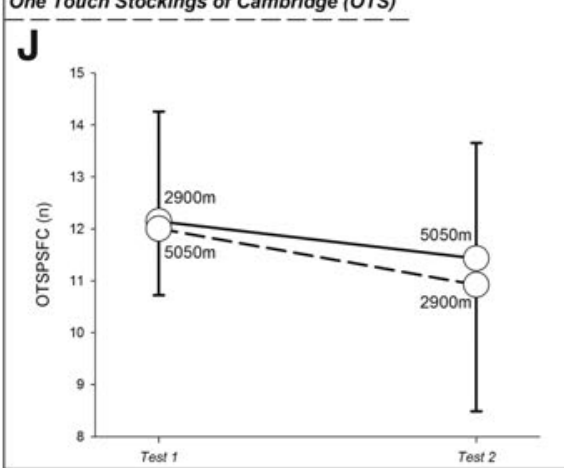

K

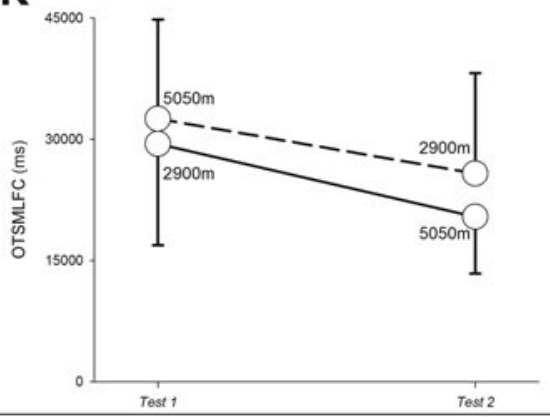

$\mathbf{L}$

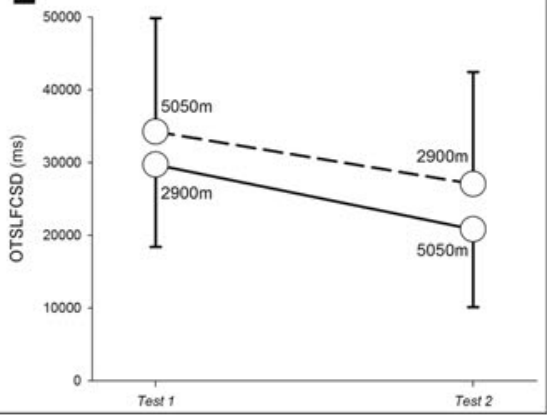


The absolute cognitive values from the two tests (Test 1 and Test 2) for both groups (MAF and $H A F)$ are presented in Figure 2 (processing speed, attention, and executive function). The figure provides uncorrected (raw) cognitive scores for both groups as a function of altitude and Test order.

\section{Effects of high-altitude exposure on cognitive functioning}

Mean performance scores on all the cognitive tests at high altitude are reported in Table 2 . The only significant Altitudeat-Test by Group interaction $\left(F_{1,17}=6.980, \eta_{p}^{2}=0.291\right.$, $p=0.017)$ was found on the RTIFMDRT. However, there was no main effect of Altitude-at-Test $\left(F_{1,17}=0.483, \eta_{p}^{2}=0.028\right.$, $p=0.496)$. Follow-up tests on the interaction did not show significant differences $\left(t_{6}=1.935, d_{\mathrm{z}}=0.733, p=0.101\right)$ in either the $M A F$ group ( $M A F$ at $2900 \mathrm{m:} 390.0 \pm 39.2 \mathrm{~ms}$ vs. $M A F$ at $5050 \mathrm{~m}: 378.2 \pm 38.8 \mathrm{~ms})$ or $H A F$ group $\left(t_{11}=-1.687\right.$, $d_{\mathrm{z}}=0.487, p=0.120, H A F$ at $5050 \mathrm{~m}: 354.4 \pm 39.7 \mathrm{~ms}$ vs. $H A F$ at $2900 \mathrm{~m}: 361.3 \pm 39.8 \mathrm{~ms}$ ). No statistically significant main effect of Altitude-at-Test or significant Altitude-at-Test by Group interaction was found for any of the other cognitive outcomes.

\section{Acclimatization effect at ALMA (5050 m) and OSF $(2900 \mathrm{~m})$}

The workers who underwent cognitive testing after 5 days of acclimatization showed better performance compared to their fellow co-workers who undertook the cognitive assessment on the day of arrival at $5050 \mathrm{~m}$ (HAF Test 1 on Day 1 vs. MAF Test 2 on Day 5). The group that had the opportunity to acclimatize showed a decrease $\left(t_{17}=-2.155, d_{\mathrm{z}}=1.086, p=0.0458\right)$ in ASTLM (ASTLM; HAF Test 1: $727.9 \pm 158.6 \mathrm{~ms}$ vs. MAF Test 2: $583.1 \pm 102.1 \mathrm{~ms})$, an increase $\left(t_{17}=2.733, d_{\mathrm{z}}=1.398\right.$, $p=0.014)$ in RVPA (RVPA, HAF Test 1: $0.89 \pm 0.06 \mathrm{~ms}$ vs. MAF Test 2: $0.96 \pm 0.03 \mathrm{~ms})$, and a decrease $\left(t_{17}=-2.375\right.$, $\left.d_{\mathrm{z}}=1.211, p=0.0296\right)$ in OTSMLFC (OTSMLFC, HAF Test 1 : $32520.6 \pm 12300.5 \mathrm{~ms}$ vs. MAF Test 2: $20388.0 \pm 7029.9 \mathrm{~ms}$ ). Similarly, the workers who performed cognitive tests after 5 days of acclimatization showed decreased variability in the cognitive function domains at ALMA $(5050 \mathrm{~m})$. There was a decrease $\left(t_{17}=-2.524, d_{\mathrm{z}}=1.282, p=0.022\right)$ in ASTLSD (ASTLSD, HAF Test 1: $257.8 \pm 84.4 \mathrm{~ms}$ vs. MAF Test $2: 168.8 \pm 50.2 \mathrm{~ms}$ ) and a similar reduction $\left(t_{17}=-2.35, d_{\mathrm{z}}=1.177, p=0.03\right)$ in RVPLSD (RVPLSD, HAF Test $1: 179.5 \pm 69.7 \mathrm{~ms}$ vs. MAF Test 2: $109.5 \pm 47.1 \mathrm{~ms})$. OTSLFCSD (OTSLFCSD, HAF Test 1: $34214.0 \pm 15637.4 \mathrm{~ms}$ vs. MAF Test 2: $20783.2 \pm 10688.3 \mathrm{~ms})$ only trended to decrease $\left(t_{17}=-2.004, d_{\mathrm{z}}=1.003, p=0.06\right)$. The acclimatization effect on the cognitive domains was not significantly different on Day 5 (HAF Test 2) compared to Day 1 (MAF Test 1) at moderate altitude (OSF, $2900 \mathrm{~m})$, except for ASTTC score, which trended to increase $\left(t_{17}=-1.84, d_{\mathrm{z}}=0.929\right.$, $p=0.083)$ after acclimatization (MAF Test $1: 155.7 \pm 1.4 \mathrm{n}$ vs. HAF Test 2: $157.4 \pm 2.2 \mathrm{n})$. The acclimatization effects for both altitude points (OSF and ALMA) are illustrated in Figure 3.

After correcting for the acute effects of $5050 \mathrm{~m}$ exposure, we found a significant improvement in processing speed (RTIFMMT), attention (ASTLM, ASTLSD, RVPA, RVPMDL, and RVPLSD), and executive function (OTSMLFC and OTSLFCSD) with acclimatization in the $M A F$ group. However, the $H A F$ group improved only in the processing speed (RTIFMDRT) domain, after removing the effects of highaltitude exposure (ALMA, $5050 \mathrm{~m}$ ), while had increased
ASTLM scores and decreased scores on the tests assessing executive functions (OTSPSFC). The altitude-corrected acclimatization effects at moderate altitude for both groups have been reported in Table 3 .

\section{Hypoxemia $\left(\mathrm{SpO}_{2}\right)$, years of work experience, and cognitive function at high altitude}

The correlation analysis between the cognitive scores for the tests conducted at high altitude (ALMA, 5050 m) and hypoxemia $\left(\mathrm{SpO}_{2}\right)$ revealed a strong positive association between $\mathrm{SpO}_{2}$ and executive functions, as measured by the OTSPSFC [OTSPSFC, $r_{(5)}=0.839, p=0.018$ ] in the $M A F$ group (Fig. 4A). There was a moderate negative association between $\mathrm{SpO}_{2}$ and variability in executive functions as measured by the OTSLFCSD [OTSLFCSD, $r_{(10)}=-0.618$, $p=0.032]$ in the $H A F$ group (Fig. 4B). There was no relationship between the cognitive scores for the tests conducted at high altitude and years of experience at high altitude.

\section{Discussion}

In this study, we did not observe statistically significant deterioration of cognitive function among high altitudeexperienced workers living near sea level during acute exposure to ALMA (5050 m) compared to exposure to moderate altitude (OSF, $2900 \mathrm{~m}$ ). The group of ALMA workers who performed the cognitive tests after 5 days of acclimatization showed significant improvements at $5050 \mathrm{~m}$ compared to their fellow co-workers who were tested immediately upon arrival. After correcting for the very high-altitude exposure effect, the acclimatization benefit was apparent only in $M A F$ group at $2900 \mathrm{~m}$. The oxygen saturation was positively correlated with executive functions in the $M A F$ group, and negatively correlated with variability in latency of executive functions among the $H A F$ group at high altitude.

The high-altitude effects observed on cognitive domains (OSF: $2900 \mathrm{~m}$ vs. ALMA: $5050 \mathrm{~m}$ ) in our study could be due to a number of factors. First, these ALMA workers were experienced high-altitude workers, with an average of $4.6 \pm 2.0$ years of repeated high-altitude work experience. Second, the study participants were highly educated (17.6 \pm 3.1 years of education), and our participants may have had higher cognitive reserve than the general population (Guerra-Carrillo et al., 2017). Third, the ALMA workers were not involved in physically demanding work (i.e., there was minimal physical exertion at altitude). A fourth explanation is that the lack of an effect could be due to the physiological acclimatization to periodic high-altitude hypoxia shown by ALMA workers. Indeed, there was a lack of significant high-altitude effect on variables such as heart rate, blood pressure, and handgrip strength (Table 1). Previous studies conducted on workers in high-altitude mining have also reported that the periodic exposure to altitude may have caused altitude acclimatization in these populations (Richalet et al., 2002; Vinnikov et al., 2016). The physiological acclimatization may have also benefited cognitive performance. The rationale for rotations within the weekly shiftwork is to minimize adverse effects of high altitude. This unique schedule that the workers have been following for years may confer some protection to them. However, the lack of baseline cognitive data at sea level or before working at ALMA, which were not possible to assess due to logistical constraints, makes it difficult to make strong conclusive 
Table 2. The Effect of Acute Altitude Exposure on the Cognitive Function (RM ANOVA) Among PerenNial ALMA (5050 M) Workers

\begin{tabular}{|c|c|c|c|c|c|}
\hline Cognitive domain & Test & Mean $\pm S D$ & $95 \% C I$ & Main effect (altitude) & Interaction (group) \\
\hline \multicolumn{6}{|c|}{ Processing speed: RTI } \\
\hline \multirow[t]{4}{*}{ RTIFMDRT (ms) } & MAF Test 1 & $390.0 \pm 39.3$ & $358.5-421.6$ & $F_{(1,17)}=0.483$ & $F_{(1,17)}=6.980$ \\
\hline & MAF Test 2 & $378.2 \pm 38.8$ & $346.7-409.7$ & $p=0.496$ & $p=0.017^{*}$ \\
\hline & HAF Test 1 & $354.5 \pm 39.7$ & $330.4-378.6$ & $\eta_{p}^{2}=0.028$ & $\eta_{p}^{2}=0.291$ \\
\hline & HAF Test 2 & $361.4 \pm 39.9$ & $337.3-385.4$ & & \\
\hline \multirow[t]{4}{*}{ RTIFMMT (ms) } & MAF Test 1 & $195.9 \pm 46.8$ & $152.7-239.1$ & $F_{(1,17)}=0.085$ & $F_{(1,17)}=1.234$ \\
\hline & MAF Test 2 & $184.0 \pm 42.8$ & $148.1-219.8$ & $p=0.774$ & $p=0.282$ \\
\hline & HAF Test 1 & $203.9 \pm 57.8$ & $170.9-236.9$ & $\eta_{n}^{2}=0.005$ & $\eta_{n}^{2}=0.068$ \\
\hline & HAF Test 2 & $210.9 \pm 46.2$ & $183.5-238.3$ & & \\
\hline \multirow[t]{4}{*}{ RTIFMTSD (ms) } & MAF Test 1 & $33.1 \pm 2.6$ & 22.9-43.4 & $F_{(1,17)}=0.002$ & 7) $=0.029$ \\
\hline & MAF Test 2 & $33.8 \pm 15.9$ & $21.3-46.3$ & $p=0.965$ & $p=0.868$ \\
\hline & HAF Test 1 & $37.3 \pm 15.9$ & $29.5-45.2$ & $\eta_{p}^{2}<0.001$ & $\eta_{p}^{2}=0.002$ \\
\hline & HAF Test 2 & $36.9 \pm 15.5$ & $27.4-46.4$ & & \\
\hline \multicolumn{6}{|l|}{ Attention: AST } \\
\hline \multirow[t]{4}{*}{ ASTTC $(n)$} & MAF Test 1 & $158.3 \pm 1.4$ & 154.7-161.9 & $F_{(1,17)}=0.25$ & $F_{(1,17)}=3.612$ \\
\hline & MAF Test 2 & $155.7 \pm 2.7$ & $153.8-157.6$ & & \\
\hline & HAF Test 1 & $155.9 \pm 5.5$ & $153.2-158.7$ & $\eta_{p}^{2}=0.015$ & $\eta_{p}^{2}=0.175$ \\
\hline & HAF Test 2 & $157.4 \pm 2.2$ & $156.0-158.9$ & & \\
\hline \multirow[t]{4}{*}{ ASTLM (ms) } & MAF Test 1 & $603.9 \pm 130.8$ & $484.8-723.1$ & $F_{(1,17)}=0.052$ & $F_{(1,17)}=0.754$ \\
\hline & MAF Test 2 & $583.1 \pm 102.1$ & $475.3-690.8$ & $p=0.822$ & $p=0.397$ \\
\hline & HAF Test 1 & $687.7 \pm 158.6$ & $596.7-778.7$ & $\eta_{p}^{2}=0.003$ & $\eta_{p}^{2}=0.042$ \\
\hline & HAF Test 2 & $699.9 \pm 150.1$ & $617.5-782.2$ & & \\
\hline \multirow[t]{4}{*}{ ASTLSD (ms) } & MAF Test 1 & $159.3 \pm 70.7$ & $95.6-223.0$ & $F_{(1,17)}=0.026$ & $F_{(1,17)}=0.379$ \\
\hline & MAF Test 2 & $168.8 \pm 50.2$ & $127.0-210.5$ & $p=0.873$ & $p=0.546$ \\
\hline & HAF Test 1 & $216.3 \pm 84.4$ & $167.7-264.9$ & $\eta_{p}^{2}=0.002$ & $\eta_{p}^{2}=0.022$ \\
\hline & HAF Test 2 & $210.8 \pm 53.5$ & $178.9-242.7$ & & \\
\hline \multicolumn{6}{|l|}{ Attention: RVP } \\
\hline \multirow[t]{4}{*}{ RVPA (ms) } & MAF Test 1 & $0.96 \pm 0.03$ & $0.92-1.00$ & $F_{(1,16)}=0.057$ & $F_{(1,16)}=0.047$ \\
\hline & MAF Test 2 & $0.96 \pm 0.03$ & $0.93-0.99$ & $p=0.814$ & $p=0.832$ \\
\hline & HAF Test 1 & $0.92 \pm 0.06$ & $0.89-0.96$ & $\eta_{p}^{2}=0.00$ & $\eta_{p}^{2}=0.003$ \\
\hline & HAF Test 2 & $0.92 \pm 0.04$ & $0.90-0.95$ & & \\
\hline \multirow[t]{4}{*}{ RVPMDL (ms) } & MAF Test 1 & $456.9 \pm 89.6$ & $338.3-575.5$ & $F_{(1,16)}=0.00005$ & $F_{(1,16)}=0.005$ \\
\hline & MAF Test 2 & $458.8 \pm 40.1$ & $415.9-501.7$ & $p=0.994$ & $p=0.944$ \\
\hline & HAF Test 1 & $497.2 \pm 173.9$ & $402.6-591.8$ & $\eta_{p}^{2}<0.001$ & $\eta_{p}^{2}<0.001$ \\
\hline & HAF Test 2 & $494.9 \pm 60.2$ & $460.7-529.1$ & & \\
\hline \multirow[t]{4}{*}{ RVPLSD (ms) } & MAF Test 1 & $122.9 \pm 74.7$ & 64.1-181.7 & $F_{(1,16)}=0.08$ & $F_{(1,16)}=0.826$ \\
\hline & MAF Test 2 & $109.5 \pm 47.1$ & $57.3-161.7$ & $p=0.782$ & $p=0.377$ \\
\hline & HAF Test 1 & $153.3 \pm 72.6$ & $106.4-200.2$ & $\eta_{p}^{2}=0.005$ & $\eta_{p}^{2}=0.049$ \\
\hline & HAF Test 2 & $160.3 \pm 73.9$ & 118.7-202 & & \\
\hline \multicolumn{6}{|c|}{ Executive function: OTS } \\
\hline \multirow[t]{4}{*}{ OTSPSFC $(n)$} & MAF Test 1 & $11.2 \pm 2.1$ & $9.9-12.5$ & 0.014 & 7) $=0.203$ \\
\hline & MAF Test 2 & $11.4 \pm 2.2$ & $9.5-13.3$ & $p=0.907$ & $p=0.658$ \\
\hline & HAF Test 1 & $11.1 \pm 1.3$ & $10.1-12$ & $\eta_{p}^{2}=0.001$ & $\eta_{p}^{2}=0.012$ \\
\hline & HAF Test 2 & $10.9 \pm 2.4$ & $9.5-12.4$ & & \\
\hline \multirow[t]{4}{*}{ OTSMLFC (ms) } & MAF Test 1 & $21793.2 \pm 12543.7$ & $11915.5-31671.0$ & $F_{(1,17)}=0.024$ & $F_{(1,17)}=0.348$ \\
\hline & MAF Test 2 & $20388.0 \pm 7029.9$ & $11750.9-29025.1$ & $p=0.879$ & $p=0.563$ \\
\hline & HAF Test 1 & $24911.1 \pm 12300.5$ & $17366.8-32455.3$ & $\eta_{n}^{2}=0.001$ & $\eta_{n}^{2}=0.020$ \\
\hline & HAF Test 2 & $25730.8 \pm 12423.6$ & $19134.1-32327.5$ & & \\
\hline \multirow[t]{4}{*}{ OTSLFCSD (ms) } & MAF Test 1 & $21849.0 \pm 11288.1$ & $10481.8-33216.2$ & $F_{(1,17)}=0.011$ & $F_{(1,17)}=0.156$ \\
\hline & MAF Test 2 & $20783.2 \pm 10688.3$ & $9677.6-31888.7$ & $p=0.918$ & $p=0.698$ \\
\hline & HAF Test 1 & $26391.0 \pm 15637.4$ & $17709.2-35072.8$ & $\eta_{p}^{2}=0.001$ & $\eta_{p}^{2}=0.009$ \\
\hline & HAF Test 2 & $27012.8 \pm 15408.7$ & $18530.8-35494.8$ & & \\
\hline
\end{tabular}

Cognitive parameters (CANTAB outcomes) during acute exposure at high altitude (ALMA, 5050 m) among perennial ALMA workers. The level of significance was set at $p<0.05$. The mean \pm SD result from the $t$-tests.

$* p<0.05$ (level of significance).

$\mathrm{CI}$, confidence interval; $F, F$-statistic; $p$, level of significance; RM ANOVA, repeated measures analysis of variance; SD, standard deviation; Test 1 , first cognitive test; Test 2, second cognitive test; $\eta_{p}^{2}$, partial eta squared (effect size).

CANTAB abbreviations: AST, Attention Switching Task; ASTLM, AST reaction Latency Mean; ASTLSD, AST reaction Latency Standard Deviation; ASTTC, AST Total Correct; OTS, One Touch Stockings of Cambridge; OTSLFCSD, OTS Latency to First Choice Standard Deviation; OTSMLFC, OTS Mean Latency First Choice; OTSPSFC, OTS Problems Solved on First Choice; RTI, Reaction Time; RTIFMDRT, RTI Median Five-choice Reaction Time; RTIFMMT, RTI Mean Five-choice Movement Time; RTIFMTSD, RTI Five-choice Movement Time Standard Deviation; RVP, Rapid Visual Processing; RVPA, RVP Accuracy; RVPMDL, RVP Mean Response Latency; RVPLSD, RVP Response Latency Standard Deviation. 

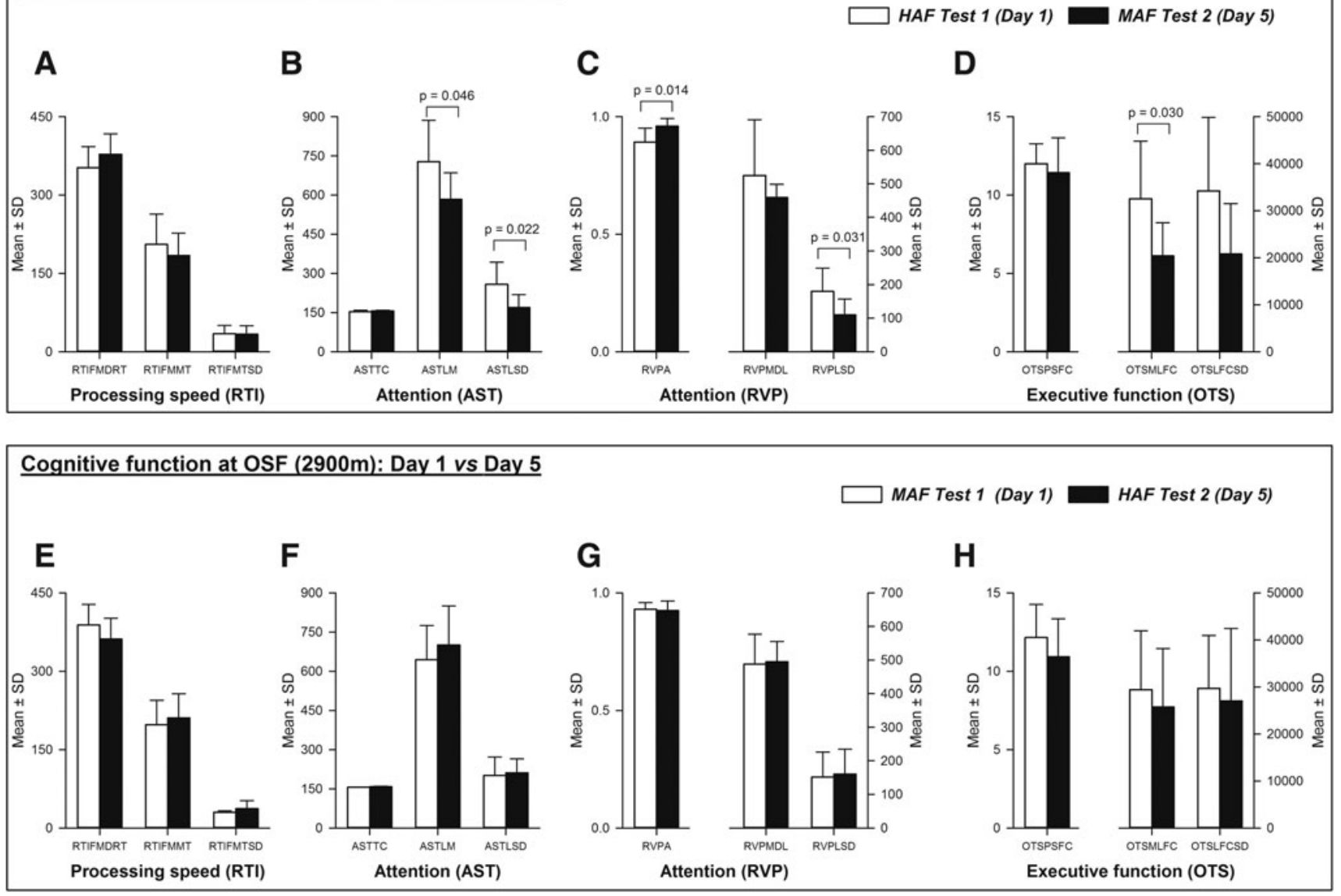

FIG. 3. Effect of high-altitude acclimatization on cognitive functions among ALMA workers. The X-axis depicts cognitive function domains and the $Y$-axis shows values of cognitive functions expressed in mean \pm standard deviation. The figure illustrates the acclimatization effect of high-altitude exposure over 5 days. The first panel (A-D) depicts cognitive functions at ALMA $(5050 \mathrm{~m})$ on the day of arrival (acute altitude effects on Day 1) and the last day of work, that is, before study participants descend for the break (acclimatization effects on Day 5). The second panel (E-H) demonstrates the influence of acclimatization on cognitive function at OSF $(2900 \mathrm{~m})$ on Day 1 (acute effects) versus Day 5 (acclimatization effects). (A, E) illustrate processing speed, that is, RTI parameters (RTIFMDRT, RTIFMMT, and RTIFMTSD). Measures of attention are represented in $(\mathbf{B}, \mathbf{F})$, the AST parameters (ASTTC, ASTLM, and ASTLSD), and (C, G), the RVP parameters (RVPA, RVPMDL, and RVPLSD). (D, H) Demonstrate executive function, showing OTS parameters (OTSPSFC, OTSMLFC, and OTSLFCSD). Symbols: Empty bars, first cognitive test on Day 1 (HAF Test 1 and MAF Test 1) at ALMA $(5050 \mathrm{~m})$ and OSF $(2900 \mathrm{~m})$ for both groups $(M A F$ and $H A F)$; solid bars, second cognitive test on Day 5 (MAF Test 2 and HAF Test 2) at OSF (2900 m) and ALMA $(5050 \mathrm{~m})$ for both groups $(M A F$ and $H A F)$. $p$, level of significance. OSF, Operation Support Facility of ALMA.

statements, and future studies should explore this further. It is possible that the cognitive performance may have been impacted by high altitude compared to sea level, but not compared to moderate altitude (OSF, $2900 \mathrm{~m}$ ). The altitude of OSF $(2900 \mathrm{~m})$, where we took the first measurements, is already an elevation that can affect performance. Previous studies have shown that ALMA workers do have impairments in cognitive functioning, which improve with oxygenation supplementation (Moraga et al., 2018).

In our study, the ALMA workers enhanced their cognitive performance at ALMA $(5050 \mathrm{~m})$ with acclimatization. The workers who performed the cognitive tests after 5 days of acclimatization performed significantly better in the tests measuring attention (decreased ASTLM and improved RPVA Accuracy) and executive functions (reduced OTSMLFC) compared to the group who was tested on the first day at $5050 \mathrm{~m}$ (Fig. 3). The acclimatized workers also demonstrated signifi- cantly less variability in attention (AST Latency Standard Deviation and RVPLSD) and tended to improve in the variability of executive functions (OTSLFCSD). These findings are consistent with previous studies that demonstrate how cognitive performance at high altitude improves with acclimatization, but these effects do not carry over upon re-ascent after a week of rest at low altitude (Subudhi et al., 2014; Pun et al., 2018a). This is opposite to the mechanisms that regulate other physiological parameters and susceptibility to acute mountain sickness (Pun et al., 2018b). It is not entirely clear why cognitive acclimatization does not follow a similar pattern as physiological acclimatization. First, it is possible that cognitive parameters are highly sensitive, and exhibit subtle changes that manifest early upon high-altitude exposure. Second, cognitive acclimatization may not yet have reached a plateau phase after 5 days. Hence, the workers may be affected every shift, and further cognitive improvement might be expected only after acclimatization for a 


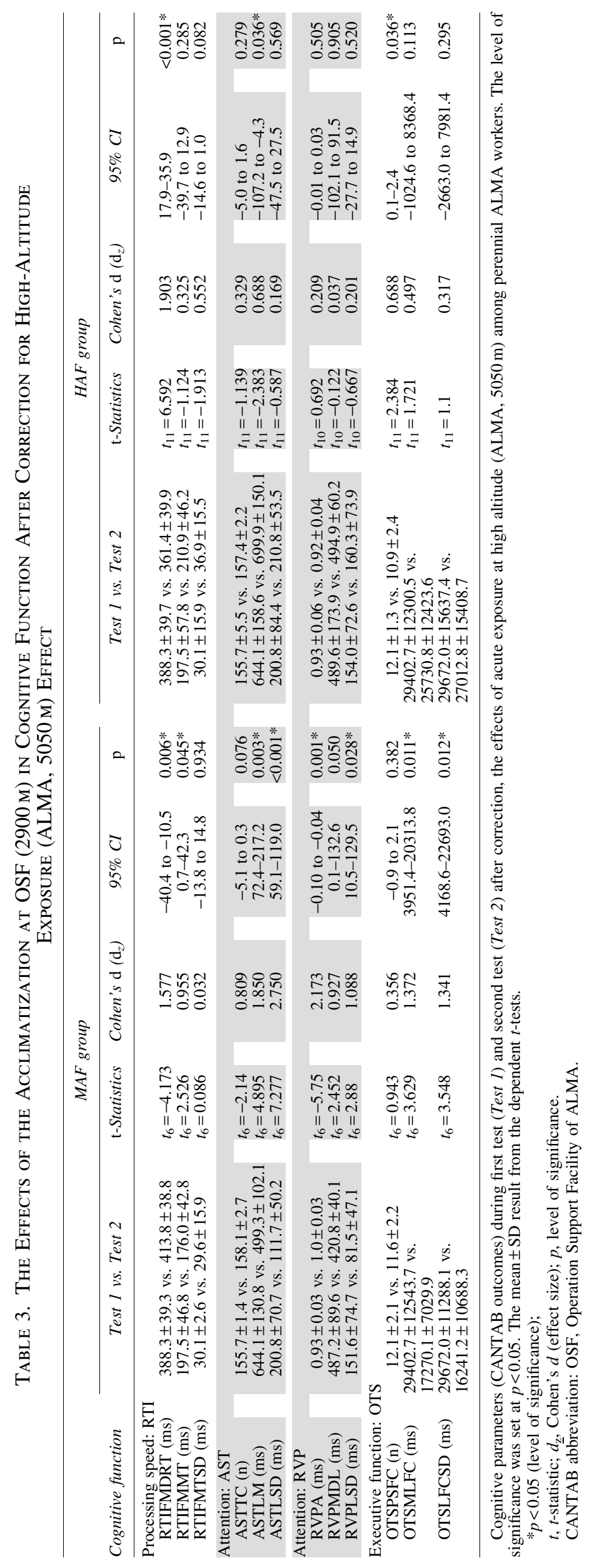



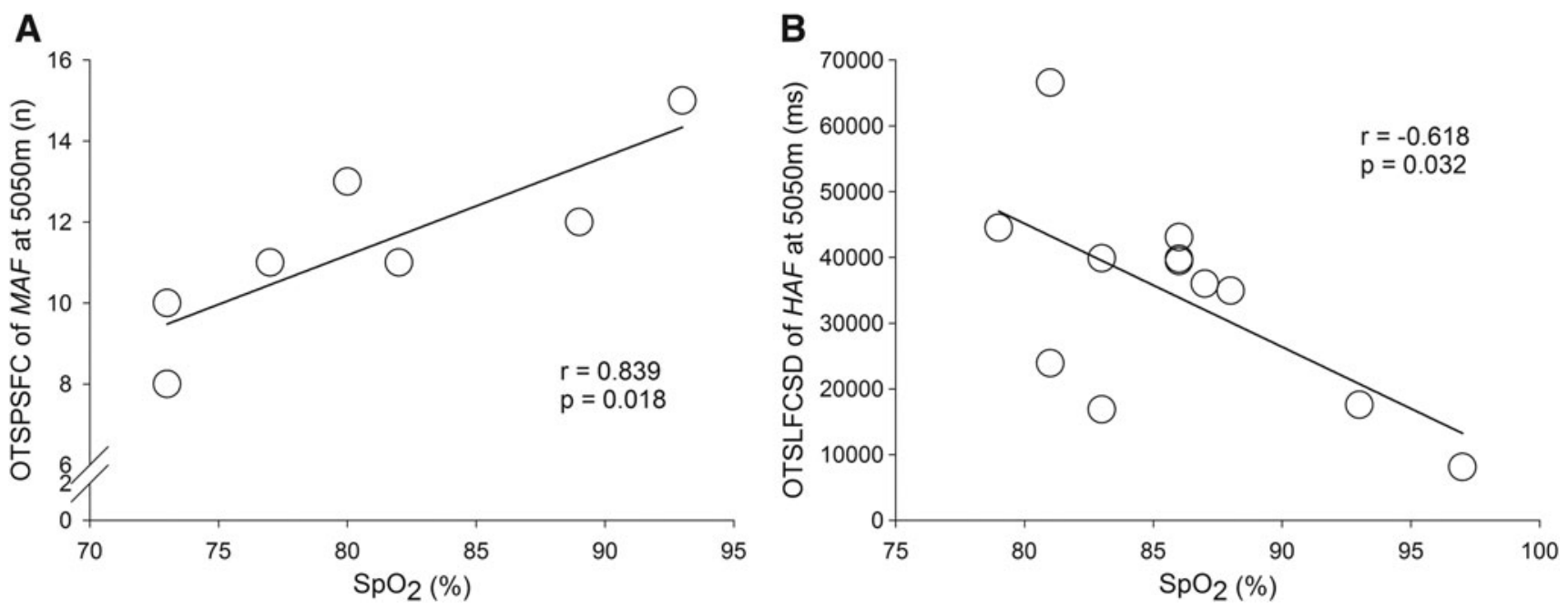

FIG. 4. Correlation of executive function domains at high altitude $(5050 \mathrm{~m})$ with blood oxygen saturation among ALMA workers. The figure illustrates correlations between executive function at high altitude (ALMA 5050 m) and blood oxygen saturation $\left(\mathrm{SpO}_{2}\right)$ among ALMA workers. The $X$-axis depicts $\mathrm{SpO}_{2}$ in percentage and $Y$-axis shows values of cognitive functions. The left panel (A) shows the positive correlation between the executive function domain $(\mathrm{OTSPSFC})$ and $\mathrm{SpO} \mathrm{O}_{2}$ at high altitude (ALMA, $5050 \mathrm{~m}$ ) among the MAF group. The right panel (B) shows the negative correlation between executive function variability (OTSLFCSD) at $5050 \mathrm{~m}$ and $\mathrm{SpO}_{2}$ among $H A F$ group. $r$, Pearson's correlation; $\mathrm{SpO} \mathrm{O}_{2}$, blood oxygen saturation.

longer period. The cognitive scores of the workers who were tested at OSF $(2900 \mathrm{~m})$ after acclimatization were not significantly different from those scores obtained upon arrival. The lack of acclimatization effect at the OSF (2900 m) suggests that the rotating work schedule benefitted the ALMA workers' cognitive performance at moderate-altitude level, but not at very high altitude. The workers may have plateaued with physiological and cognitive acclimatization by sleeping at this moderate altitude and working at ALMA on a shift basis. On the other hand, the moderate altitude (OSF, $2900 \mathrm{~m}$ ) may not have had a significant effect on cognitive performance and hence, there was little room to improve with 5 days of acclimatization. Interestingly, after correcting for the effects of $5050 \mathrm{~m}$ exposure, the $M A F$ group performed better during their second cognitive test at the OSF $(2900 \mathrm{~m})$, that is, after acclimatization. However, we did not see similar acclimatization effects in the $H A F$ group, except with respect to reaction time (RTIFMDRT). In fact, $H A F$ participants performed worse on attention tasks (ASTLM) and executive functions (OTSPSFC) tasks during the second cognitive assessment. These findings, and especially the $M A F$ acclimatization effects at $2900 \mathrm{~m}$, should be carefully interpreted and warrant further investigation with a larger sample size in a similar setting, with multiple testing time points over the period of high-altitude stay, especially among high-altitude workers.

As expected, the participants in both groups were significantly hypoxemic with exposure to high altitude (Table 1) and there was a strong association between $\mathrm{SpO}_{2}$ at high altitude and individuals' cognitive performance at $5050 \mathrm{~m}$, particularly for the executive function domain. The participants with higher $\mathrm{SpO}_{2}$ had better executive functions within the $M A F$ group, while worse $\mathrm{SpO}_{2}$ led to higher variability in executive latency within the $H A F$ group (Fig. 4). In fact, these findings are in line with the findings from Moraga et al. (2018), who reported beneficial effects of oxygen supplementation among ALMA workers leading to improvements in some of the assessed cognitive domains. Our findings, together with the ones reported by Moraga et al., support the long-standing advocacy for oxygen enrichment in high- altitude workstations as well as for permanent dwellers, to improve overall performance (West 2016a, b). The ALMA workers engage in technically demanding scientific and engineering tasks, which involve manipulation of extremely delicate instrumentation: their workplace performance may benefit from oxygen enrichment in the work facility. The number of years working at ALMA did not seem to be associated with the cognitive changes.

It is intriguing that the $H A F$ group had slightly higher $\mathrm{SpO}_{2}$ (measured on the first day of the shift-work week at $5050 \mathrm{~m}$ ) compared to $\mathrm{SpO}_{2}$ of $M A F$ group (measured at the end of the of the shift-work week). This difference, however, was not statistically significant. This $\mathrm{SpO}_{2}$ difference could be due to individual variability in our relatively small sample size. Second, the $\mathrm{SpO}_{2}$ measured with pulse oximetry can be at times unreliable (Luks and Swenson, 2011), and it may not always reflect clinical presentation at altitude (Chen et al., 2012; Leichtfried et al., 2016). The hypoxemia reflected with long-term monitoring of $\mathrm{SpO}_{2}$ seems to be better at assessing acute clinical presentation (Mandolesi et al., 2014) and this may hold true for cognitive functions as well.

\section{Strengths and limitations}

Strengths. This is the first study using a comprehensive cognitive battery to test the effects of high altitude on cognition among high-altitude workers who have been doing this type of shift-work for many years. The study design has enabled us to elucidate not only the effect of altitude but also the effect of acclimatization at both altitude points $(2900$ and $5050 \mathrm{~m})$. The cohort of workers that we recruited had been shift-working for several years and were employed in intellectually demanding professional tasks at high altitude. The custom cognitive battery selected from the CANTAB collection was chosen due to portability and robustness. The CANTAB tests have been validated for use across multiple languages, and were therefore suitable for our study participants who were native Spanish speakers. 
Limitations. Our sample size is small, although hundreds of individuals are currently working at ALMA. We sampled as many participants as we could within the constraints (e.g., time, subject recruitment, research personnel, and equipment) presented to us. As a result, we did not perform an a priori power calculation. Unfortunately, the study participants were not equally distributed in the two groups and one participant dropped out from the $M A F$ group, which was already smaller at the beginning. Furthermore, the Altitude-at-Test and the test order were not manipulated factorially and this forced us to compute corrected scores to isolate the effects of altitude from the effects due to test repetition. Despite the corrections and robustness of the cognitive battery, we cannot rule out the fact that our results may have been confounded by the order of tests, that is, repetition effects at high altitude. The participants in our study are all males. Unfortunately, we were unable to enroll women in the study due to time constraints in the recruitment phase and the fact that the majority of high-altitude workers at the ALMA facility are males.

Similarly, we were unable to collect baseline data from near sea level due to logistic constraints. Moreover, we collected general information on participant medical history, smoking habits, and alcohol and caffeine consumption, but we did not specifically record their use immediately before cognitive testing. The use of stimulants (nicotine and caffeine) may have influenced the workers' performance on the cognitive tests. These are important limitations of this study; however, recruitment and scheduling issues are common in field studies on very specific populations that unfortunately lack the rigor of laboratory-conducted experimental studies. Future studies should address these issues. Our participants are highly educated and follow a unique pattern of highaltitude acclimatization; therefore, our findings may not be generalizable to other high altitude sojourners such as occasional trekkers or climbers.

\section{Conclusion}

The ALMA workers seem to have benefited from the periodic weekly alternating work-shift cycle for several years at high altitude as observed with the minimal impairments in their cognitive functioning during acute exposure to $5050 \mathrm{~m}$ compared to $2900 \mathrm{~m}$. However, the workers significantly improved on their cognitive performances with acclimatization. Similarly, the changes in executive functions domain were associated with hypoxemia during acute exposure to $5050 \mathrm{~m}$. These findings highlight the importance of acclimatization on cognitive functioning even among perennial high-altitude workers at very high altitude, and further emphasize the importance of oxygen enrichment as reported by Moraga et al. (2018). Acclimatization and oxygen enrichment could potentially maximize the performance of workers at ALMA and at the same time reduce the errors and risks by improving blood oxygen level. A gradual ascent in the first couple of days with less demanding working hours spent at ALMA could mitigate the effects of ascent on cognitive ability. Future research is required to establish an optimal acclimatization strategy in these settings and with this work schedule.

\section{Acknowledgments}

We express our sincere thanks to the ALMA workers who took part in our study during their work-shift cycle at the
ALMA Observatory, Chile. The research would have been impossible without their involvement and cooperation throughout the study period. We would also like to acknowledge our Chilean collaborators from the ALMA Head Office in Santiago, Chile, and the staff of the ALMA observatory, who greatly facilitated the study. Finally, we would like to thank Alicia Morales Soto for helping facilitate the logistics in Santiago during our study expedition.

\section{Disclaimer}

The content of the information does not necessarily reflect the position or the policy of the Government, and no official endorsement should be inferred.

\section{Author Disclosure Statement}

No competing financial interests exist.

\section{Funding Information}

M.J.P. is supported by a Discovery Grant from the Natural Sciences and Engineering Research Council (NSERC) of Canada (Principal Investigator [PI], M.J.P.; 2014-05554), and a CIHR Operating Grant on the Regulation of cerebral blood flow in OSA (PI: M.J.P.). S.E.H. received support from the Dr. Chen Fong doctoral scholarship (Hotchkiss Brain Institute). L.L.D. is supported by an Alberta Innovates Health Services (AIHS) Postgraduate Fellowship. V.G. is supported by The Brenda Strafford Centre on Aging, within the O'Brien Institute for Public Health, the Brenda Strafford Foundation Chair for Alzheimer Research (BSFCAR), and the Alzheimer Society of Canada Postdoctoral Fellowship. M.J.P. holds the BSFCAR. K.E.B. and S.U. are supported by Lunge Zurich, Swiss National Science Foundation. B.G. is supported by the Institute for Collaborative Biotechnologies through grant W911NF-09-0001 from the U.S. Army Research Office.

\section{References}

Asmaro D, Mayall J, and Ferguson S. (2013). Cognition at altitude: Impairment in executive and memory processes under hypoxic conditions. Aviat Space Environ Med 84:1159-1165.

Bjursten H, Ederoth P, Sigurdsson E, Gottfredsson M, Syk I, Einarsson O, and Gudbjartsson T. (2010). S100B profiles and cognitive function at high altitude. High Alt Med Biol 11: 31-38.

CANTAB. (1994). Cambridge Neuropsychological Test Automated Battery (CANTAB). Cambridge Cognition Ltd, Company No. 4338746, UK.

Chen HC, Lin WL, Wu JY, Wang SH, Chiu TF, Weng YM, Hsu TY, and Wu MH. (2012). Change in oxygen saturation does not predict acute mountain sickness on Jade Mountain. Wilderness Environ Med 23:122-127.

Cramer NP, Korotcov A, Bosomtwi A, Xu X, Holman DR, Whiting K, Jones S, Hoy A, Dardzinski BJ, and Galdzicki Z. (2019). Neuronal and vascular deficits following chronic adaptation to high altitude. Exp Neurol 311:293-304.

Davranche K, Casini L, Arnal PJ, Rupp T, Perrey S, and Verges S. (2016). Cognitive functions and cerebral oxygenation changes during acute and prolonged hypoxic exposure. Physiol Behav 164:189-197.

Guerra-Carrillo B, Katovich K, and Bunge SA. (2017). Does higher education hone cognitive functioning and learning efficacy? Findings from a large and diverse sample. PLoS One 12:e0182276. 
Hornbein TF. (2001). The high-altitude brain. J Exp Biol 204: 3129-3132.

Kramer AF, Coyne JT, and Strayer DL. (1993). Cognitive function at high altitude. Hum Factors 35:329-344.

Leichtfried V, Basic D, Burtscher M, Gothe RM, Siebert U, and Schobersberger W. (2016). Diagnosis and prediction of the occurrence of acute mountain sickness measuring oxygen saturation-Independent of absolute altitude? Sleep Breath 20:435-442.

Limmer M, and Platen P. (2018). The influence of hypoxia and prolonged exercise on attentional performance at high and extreme altitudes: A pilot study. PLoS One 13:e0205285.

Lowe C, and Rabbitt P. (1998). Test/re-test reliability of the CANTAB and ISPOCD neuropsychological batteries: Theoretical and practical issues. Cambridge Neuropsychological Test Automated Battery. International Study of PostOperative Cognitive Dysfunction. Neuropsychologia 36:915923.

Luks AM, and Swenson ER. (2011). Pulse oximetry at high altitude. High Alt Med Biol 12:109-119.

Maa EH. (2010). Hypobaric hypoxic cerebral insults: The neurological consequences of going higher. NeuroRehabilitation 26:73-84.

Mandolesi G, Avancini G, Bartesaghi M, Bernardi E, Pomidori L, and Cogo A. (2014). Long-term monitoring of oxygen saturation at altitude can be useful in predicting the subsequent development of moderate-to-severe acute mountain sickness. Wilderness Environ Med 25:384-391.

McMorris T, Hale BJ, Barwood M, Costello J, and Corbett J. (2017). Effect of acute hypoxia on cognition: A systematic review and meta-regression analysis. Neurosci Biobehav Rev 74:225-232.

Moraga FA, Lopez I, Morales A, Soza D, and Noack J. (2018). The effect of oxygen enrichment on cardiorespiratory and neuropsychological responses in workers with chronic intermittent exposure to high altitude (ALMA, 5,050 m). Front Physiol 9:187.

Niedermeier M, Weisleitner A, Lamm C, Ledochowski L, Fruhauf A, Wille M, Burtscher M, and Kopp M. (2017). Is decision making in hypoxia affected by pre-acclimatisation? A randomized controlled trial. Physiol Behav 173:236-242.

Ochi G, Kanazawa Y, Hyodo K, Suwabe K, Shimizu T, Fukuie T, Byun K, and Soya H. (2018a). Hypoxia-induced lowered executive function depends on arterial oxygen desaturation. J Physiol Sci 68:847-853.

Ochi G, Yamada Y, Hyodo K, Suwabe K, Fukuie T, Byun K, Dan I, and Soya H. (2018b). Neural basis for reduced executive performance with hypoxic exercise. Neuroimage 171: 75-83.

Pun M, Guadagni V, Bettauer KM, Drogos LL, Aitken J, Hartmann SE, Furian M, Muralt L, Lichtblau M, Bader PR, Rawling JM, Protzner AB, Ulrich S, Bloch KE, Giesbrecht B, and Poulin MJ. (2018a). Effects on cognitive functioning of acute, subacute and repeated exposures to high altitude. Front Physiol 9:1131.

Pun M, Hartmann SE, Furian M, Dyck AM, Muralt L, Lichtblau M, Bader PR, Rawling JM, Ulrich S, Bloch KE, and Poulin MJ. (2018b). Effect of acute, subacute, and repeated exposure to high altitude $(5050 \mathrm{~m})$ on psychomotor vigilance. Front Physiol 9:677.

Richalet JP, Donoso MV, Jimenez D, Antezana AM, Hudson C, Cortes G, Osorio J, and Leon A. (2002). Chilean miners commuting from sea level to $4500 \mathrm{~m}$ : A prospective study. High Alt Med Biol 3:159-166.

Subudhi AW, Bourdillon N, Bucher J, Davis C, Elliott JE, Eutermoster M, Evero O, Fan JL, Jameson-Van Houten S, Julian CG, Kark J, Kark S, Kayser B, Kern JP, Kim SE, Lathan C, Laurie SS, Lovering AT, Paterson R, Polaner DM, Ryan BJ, Spira JL, Tsao JW, Wachsmuth NB, and Roach RC. (2014). AltitudeOmics: The integrative physiology of human acclimatization to hypobaric hypoxia and its retention upon reascent. PLoS One 9:e92191.

Syvaoja HJ, Tammelin TH, Ahonen T, Rasanen P, Tolvanen A, Kankaanpaa A, and Kantomaa MT. (2015). Internal consistency and stability of the CANTAB neuropsychological test battery in children. Psychol Assess 27:698-709.

Vinnikov D, Brimkulov N, and Krasotski V. (2016). Chronic intermittent hypoxia and blood pressure: Is there risk for hypertension in healthy individuals? High Alt Med Biol 17: 5-10.

West JB. (2016a). Barcroft's bold assertion: All dwellers at high altitudes are persons of impaired physical and mental powers. J Physiol 594:1127-1134.

West JB. (2016b). Oxygen conditioning: A new technique for improving living and working at high altitude. Physiology (Bethesda) 31:216-222.

Wilson MH, Newman S, and Imray CH. (2009). The cerebral effects of ascent to high altitudes. Lancet Neurol 8:175-191.

Address correspondence to:

Marc J. Poulin, PhD, DPhil

Department of Physiology and Pharmacology Hotchkiss Brain Institute

Cumming School of Medicine University of Calgary

HMRB-210, 3330 Hospital Drive NW T2N 4N1 Calgary Canada

E-mail: poulin@ucalgary.ca

Received February 6, 2019; accepted in final form July 4, 2019. 